Revue d'histoire de l'Amérique française

REYUE D.HISTOIRE DE L'AMÉRIQUE FRANÇAISE

\title{
Un Canadien gouverneur du Sénégal
}

\section{L ouis Le Gardeur de Repentigny, 1721-1786 (suite)}

\section{Jore}

Volume 15, numéro 3, décembre 1961

URI : https://id.erudit.org/iderudit/302136ar

DOI : https://doi.org/10.7202/302136ar

Aller au sommaire du numéro

Éditeur(s)

Institut d'histoire de l'Amérique française

ISSN

0035-2357 (imprimé)

1492-1383 (numérique)

Découvrir la revue

Citer cet article

Jore, L. (1961). Un Canadien gouverneur du Sénégal : L ouis Le Gardeur de Repentigny, 1721-1786 (suite). Revue d'histoire de l'Amérique française, 15(3), 396-418. https://doi.org/10.7202/302136ar d'utilisation que vous pouvez consulter en ligne.

https://apropos.erudit.org/fr/usagers/politique-dutilisation/ 


\title{
UN CANADIEN GOUVERNEUR DU SÉNEGAL * Louis Le Gardeur de Repentigny
}

\author{
1721-1786 \\ (suite) \\ VI
}

\section{RAPPEL DE LOUIS DE REPENTIGNY}

Lorsqu'il avait été désigné pour occuper le poste de Gouverneur des Établissements français de la Côte d'Afrique, Repentigny avait été chargé de procéder de concert avec l'Ordonnateur Bailly, à une enquête sur les faits reprochés à son prédécesseur, Mr. Dumontet, ainsi qu'à certains officiers militaires et civils. Le ministre leur avait remis des Instructions confidentielles relatives à cette mission et leur avait fait savoir qu'ils étaient dispensés dans son accomplissement, de suivre strictement les règles prescrites par la Règlementation de la Procédure Criminelle puisqu'il n'existait pas de tribunaux réguliers dans la Colonie.

Cette dérogation aux règles en vigueur constituait déjà une décision des plus regrettables, elle s'aggravait du fait qu'il est toujours fâcheux de confier au remplaçant d'une personne pourvue d'un emploi, quel qu'il soit, de contrôler les agissements de son prédécesseur. Pour n'avoir pas mené cette enquête avec énergie et s'être comporté, il faut le reconnaître, de façon peu adroite, Louis Le Gardeur de Repentigny fut sévèrement blâmé par le chef du Département de la Marine et des Colonies.

J'ai reçu, Monsieur, lui écrivit le ministre le 10 octobre 1784, les lettres par lesquelles vous m'avez rendu compte des opérations que vous avez faites avec Mr. Bailly pour remplir la commission dont vous étiez chargés en commun pour vérifier les faits

* Voir notre Revue, XV : 64-89, 256-276. 
imputés tant au Gouverneur qu'aux officiers d'administration, et Mr de la Jaille m'a remis les informations que vous lui aviez confiées. La présence de Mr. Dumontet a dû gêner la déposition et je suis étonné qu'après lui avoir demandé des explications, que vous ayez jugées nécessaires, d'après les pièces qui vous avaient été remises, vous ne l'ayez pas fait partir pour France afin de travailler avec plus de liberté. Ce parti vous était indiqué par le renvoi qu'il avait fait lui-même des officiers d'administration qui étaient en quelque sorte ses parties et vous avez dû regretter de ne pas l'avoir pris lorsque vous l'avez vu frapper en votre présence un témoin à la déposition duquel il ne devait pas être appelé. Quoi qu'il en soit j'ai chargé une personne de confiance de l'examen de cette affaire pour me mettre en état de prendre les ordres du Roy.

Je n'ai pu voir qu'avec peine les imputations que vous avez faites par une de vos lettres à Mr. Bailly et les plaintes que ce dernier m'a également adressées. Il en résulte qu'il n'a pas régné entre vous le concert et l'union qui vous avaient été recommandées. Votre collègue avait à se défendre de la prévention envers ses confrères; vous étiez dans le même cas envers Mr. Dumontet qui d'ailleurs était présent. Je vous transmettrai au surplus la décision du Roy lorsqu'il se sera prononcé.

Douze jours après l'expédition de la lettre précédente, le ministre la complétait par une seconde ainsi conçue:

Vous avez marqué, Monsieur, par votre lettre du 28 février dernier No 1, que Mr. Dumontet vous aïant requis de renvoyer en France le sieur Aubert, Greffier de la Colonie, vous avez cru devoir le faire partir pour la France.

Cet employé m'ayant demandé la communication des griefs qui pouvaient avoir occasionné sa destitution et son renvoi de la colonie afin de se justifier, j'ai écrit à Mr. Dumontier (sic) pour en être informé. Cet ancien Gouverneur est entré dans des détails sur la succession Gondreville dans les affaires de laquelle il prétend que le sieur Aubert s'est rendu suspect de connivence, ou du moins de négligence impardonnable, ce qui l'avait porté à lui retirer sa confiance et à ne plus l'employer dans le secrétariat. 
Il ajoute qu'il était naturel que vous en usassiez de même et que vous le renvoyez en France, comme n'étant pourvu d'aucun brevet du Roy. Vous voyez que c'est à vous que Mr. Dumontier (sic) attribue le renvoi du sieur Aubert qui jouissant dans ses deux places de l'agrément du Roy consigné dans une dépêche adressée à Mr. Dumontier (sic) et Daigremont le 30 Décembre 1783 , ne pouvait être déplacé sans des motifs valables que je ne trouve pas dans la réponse de Mr. Dumontier (sic). Je ne puis pas, en conséquence, approuver la complaisance qui vous a porté à user contre lui de la voie de l'autorité.

Une autre dépêche du 22 octobre 1784 refusait de remplacer le sieur Aubert par un certain Anteline, choisi sur place, ne considérant pas sa place comme vacante.

L'affaire Dumontet fut examinée par des Conseillers d'État et un Maître des Requêtes rapporteur. Les conclusions présentées au Conseil des Dépêches par rapport du 9 mars 1785 valurent à l'ancien Gouverneur Dumontet d'être exclu de tout emploi et au nouveau, Louis de Repentigny, qui s'était laissé aller à une telle partialité, de ne plus conserver la confiance du Gouvernement.

«On pense toutefois, concluait l'avis des Conseillers dÉtat, qu'il convient de mettre une distance entre l'exécution des articles ci-dessus et de la destitution du sieur de Repentigny afin de ne pas le confondre avec ceux dont la conduite a été plus répréhensible; il est un homme d'honneur, mais très impropre à une administration. 》

Ce fut le 8 octobre 1785 que le ministre fit savoir à Repentigny qu'il avait été remplacé. La Dépêche était ainsi conçue:

Le Roy aïant jugé à propos, Monsieur, de nommer au Gouvernement du Sénégal, le Chevalier de Boufflers, Maréchal de Camp, je vous prie de faire les dispositions nécessaires pour lui remettre l'administration dont vous êtes chargé. Il partira dans quatre à cinq semaines pour se rendre à destination. Je vous informerai alors des mesures qui auront été 
prises pour votre retour; je mettrai au surplus avec plaisir sous les yeux de Sa Majesté, vos anciens services et je serai fort aise de vous procurer les grâces dont ils vous rendront susceptibles.

J'ai l'honneur d'être parfaitement, Monsieur, votre très humble et très obéissant serviteur. ${ }^{80}$

Cette communication fut suivie le 19 du même mois par la suivante:

Je vous ai prévenu, Monsieur, que le Roy avait nommé Mr. le Chevalier de Boufflers au Gouvernement du Sénégal. Cet officier doit se rendre à Rochefort pour passer à sa destination sur la corvette «Le Rossignol 》. Je vous prie de lui remettre à son arrivée les détails du commandement dont vous êtes chargé.

La Corvette «Le Rossignol », affectée à la station du Sénégal, demeurera sur les côtes, mais j'ai donné ordre d'expédier la flutte (sic) du Roy, « La Bretonne » qui prendra à Lorient et à Rochefort des recrues et des effets qu'elle transportera au Sénégal. Ce bâtiment est, au surplus destiné à vous ramener en France.

Je mettrai avec plaisir à votre retour vos services sous les yeux du Roy pour vous procurer les grâces de Sa Majesté.

J'ai l'honneur, etc.

Bien qu'il fût très heureux de sa nomination à la tête des Etablissements du Sénégal, le Chevalier de Boufflers souffrait de la disgrâce de son prédécesseur. Il accomplit le geste chevaleresque et digne d'un gentilhomme de plaider la cause de son camarade malheureux auprès du ministre auquel il écrivit de Rochefort le 29 novembre 1785 :

Monseigneur,

J'ai l'honneur de vous adresser différentes lettres dont les réponses pourront, selon toute apparence, me parvenir encore dans ce pays-cy car le vent qui avait été constamment au Nord-Est s'est

${ }^{80}$ Louis de Repentigny avait écrit des « Mémoires 》 qu'il prêta à Golberry et dont ce dernier reconnaît avoir fait usage pour la rédaction de son livre: Fragments d'un voyage en Afrique (Paris, 1802, pages 11 et 12). Ces mémoires sont vraisemblablement perdus. 
retourné subitement à mon arrivée et paraît devoir tenir longtemps dans la partie de l'Ouest.

J'ai vu « Le Rossignol » sur lequel je dois passer; il est d'une petitesse extrême et je me vois obligé de laisser ici la majeure partie de mon monde et de mes effets attendre l'arrivée de la flûte « La Bretonne »; mais le manque de recrues à Lorient sera encore une nouvelle cause de retard à moins que vous n'accordiez la demande que j'ai l'honneur de vous faire de tenir mon complément du fonds $d u$ corps de Lorient. Cette disposition nuirait peu à ce corps qui aurait bientôt réparé ses pertes et serait très utile au bataillon d'Affrique (sic) qui a besoin d'artilleurs et d'ouvriers d'état. Songés, Monseigneur, que d'une part nous ne pouvons pas nous passer d'hommes et que, de l'autre, nous ne pouvons pas attendre longtemps «La Bretonne » qui doit ramener Mr. de Repentigny. Je fais attendre Mr. Blanchot ${ }^{81}$ à Rochefort pour ne pas le mettre longtemps en présence avec $\mathrm{Mr}$. Deladevèze ${ }^{82}$ dont je crains la mauvaise tête, et je tâcherai de renvoyer ce dernier par un bateau de commerce pour épargner à Mr. de Repentigny le chagrin de voyager ensemble. Je ne sçaurais assez me louer de Mr. de Vaudreuil et de Mr. de la Grandville ainsi que de Mr. le Chevalier de Brache avec lequel je suis sûr de m'entendre parfaitement.

Il ne me reste, Monseigneur, qu'à vous supplier à l'avance de traiter favorablement $\mathrm{Mr}$. de Repentigny. Tout ce que j'ai vu et tout ce que j'apprends sur son compte annonce le meilleur et le plus brave homme du monde; il est couvert de blessures et a de très beaux et très longs services, et s'il a commis des fautes, elles sont toutes excusables et ne doivent pas empêcher un ancien brigadier de devenir Maréchal de Camp à la première promotion et de jouir

81 Le Commandant Blanchot avait été nommé major du Bataillon d'Afrique et second du Chevalier de Boufflers, son ancien colonel au Régiment de Chartres.

82 Précédemment major du Bataillon d'Afrique qui avait suscité mille difficultés à Repentigny. Les dossiers recueillis par Moreau de St-Méry (Archives de France F3 61) contiennent des documents qui témoignent hautement du déséquilibre mental de cet officier, ou tout au moins du plus fâcheux caractère. 
d'une retraite honorable. Je serais très heureux, Monseigneur, si ma prière était écoutée et je garderais tout ce que vous feriés pour Mr. de Repentigny comme une récompense anticipée des services que j'essaierai de rendre à la colonie.

Arrivé à St-Louis le 15 janvier 1786, Boufflers écrivait à son oncle le Maréchal de Beauvau le 6 mars: "Vous voulez savoir avec qui et comment je passe ma vie. J'aurais de la peine à vous en faire un tableau parce que d'ici au départ de $\mathrm{Mr}$. de Repentigny, je ne puis pas prendre une certaine assiette et que ce départ se diffère tous les jours, tant par les obstacles qu'il cherche, dit-on, à y mettre pour des raisons d'intérêt qu'il n'ose pas dire ${ }^{83}$ et que je n'ose pas soupçonner, que par l'impossibilité de passer la barre qui, depuis quinze jours, a rompu de nouveau toute communication entre l'île et la rade ... En attendant, cette pauvre flûte est près de manquer d'eau ...; si cette séparation forcée durait encore quinze à vingt jours, ce bâtiment serait obligé d'aller sans pouvoir m'en demander l'ordre, faire son eau à Gorée pour revenir ici achever son débarquement et prendre Mr. de Repentigny... J'ai ordinairement quatorze à quinze couverts et tant que Mr. de Repentigny restera ici, je n'en aurai pas moins ... Le commandant de la troupe est logé chez moi et l'ordonnateur y mange en attendant qu'ils aient chacun leur maison, mais tant que Mr. de Repentigny sera ici, il y aura engorgement. »

Dans une lettre datée du 27 mars, Boufflers écrivait: « Je crois, et même je suis presque sûr que Mr. de Repentigny doit partir demain par la Bayonnaise. » Prévision qui ne dut pas se réaliser puisque son prédécesseur n'arriva à Lagos, au Portugal, qu'un peu avant le 28 mai, date à laquelle celui-ci annonçait

83 Il est assez singulier que Repentigny se soit mêlé lui aussi d'affaires commerciales. Une lettre de Boufflers fait allusion à une cargaison de captifs noirs : « 20 janvier (?) J'apprends dans ce moment que ce $\mathrm{Mr}$. Blondeau qui doit te porter de mes nouvelles et qui devait prendre à St-Domingue le premier bâtiment allant en France, n'en fera peut-être rien. Il mène environ cent nègres achetés, dit-on, entre lui et $\mathrm{Mr}$. de Repentigny et ne sortira pas de St-Domingue qu'ils ne soient vendus jusqu'au dernier. 》 S'agissaitil d'un prêt d'argent consenti par Repentigny à ce Blondeau ? Mais peutêtre ce prêt n'avait-il été consenti par Repentigny que lorsqu'il n'était plus chef de la colonie. 
par une lettre son départ de Gorée dans un déplorable état de santé qui lui faisait penser qu'il n'arriverait pas vivant en Europe. En raison du mauvais temps, la Bayonnaise n'avait pu entrer dans le port de Lisbonne. «Ma santé, poursuivait Repentigny, s'affaiblit tous les jours; ne pouvant gagner Vigo où j'aurais désiré d'aller de préférence, Mr. de Guerpel a été forcé de venir à Lagos. Le Commandant du navire trouve appui empressé dans les agens du Gouvernement et l'aide de Jean-Marie Dominguet, fils d'un Français natif de Lion (sic), jeune négociant établi dans cette ville où il est marié depuis quatre ans. Le Portugais chargé des affaires des Français n'a pas paru. »

En raison des lenteurs de communication de l'époque, Repentigny n'était pas encore arrivé à Paris le 20 juin date à laquelle Mme de Sabran écrivait à Boufflers: "Comment pourrais-je me rendre raison du retard du vaisseau de $\mathrm{Mr}$. de Repentigny qui devait être ici depuis trois mois ? » et ce n'est que le 12 juillet que dans une autre lettre elle mandait à Boufflers: «Enfin, je commence à respirer, mon bien cher mari, Mr. de Castries vient de recevoir une lettre de Mr. de Repentigny datée du Portugal. Je ne sais pas pourquoi il a pris cette route car elle ne me paraît pas la plus courte, mais enfin sa lettre est remplie de tes éloges. »

Repentigny avait été précédé par plusieurs lettres de Boufflers demandant qu'on lui vînt en aide. L'une d'elle, sans date ni nom de destinataire s'exprimait ainsi: "Vous verrez Mr. de Repentigny, je le recommande à vos soins et à ceux de tous mes parents et de tous mes amis. Je vous ai déjà écrit à son sujet; je répète et je redouble toutes mes sollicitations; Tout ce que l'on fera pour lui, je le recevrai à titre de récompense et je regarderais comme une tache à ma vie d'avoir pu causer, quoique bien innocemment, le malheur d'un digne homme et de n'avnir pas pu contribuer à sa consolation. Dites à qui il appartiendra, à mon oncle, ${ }^{84}$ à mon ami le Chevalier de Beauteville, ${ }^{85}$ à l'Évêque

84 Le Maréchal de Beauvau.

85 Pierre du Buisson, Chevalier de Beauteville (1703-1790). Après une belle carrière militaire il avait été promu Lieutenant Général en 1762. Nommé ensuite ambassadeur en Suisse il conserva ce poste jusqu'en 1775. 
d'Arras, ${ }^{86}$ à Madame de Lauzun ... ${ }^{87}$ qu'il faudrait que Mr. de Repentigny fût Maréchal de Camp avec six mille livres de pension. Il a eu plus de malheurs que de torts; il est couvert de blessures; il a près de cinquante ans de services; il est adoré dans ce pays-ci ; tout cela parle pour lui. Je sais qu'il est protégé par des gens que $\mathrm{Mr}$. le Maréchal ${ }^{88}$ n'aime pas, mais c'est une raison de plus auprès de Mr. le Maréchal qui fera par générosité ce qu'un autre ferait par faiblesse. »

Dans une autre lettre, arrivée en même temps que la précédente, Boufflers écrivait: «Faites à Mr. de Repentigny et faites lui faire l'accueil que nous lui devons tous. Hélas! il m'a vu sans doute arriver avec peine, et je le vois partir avec envie. Il verra tout ce que je regrette; il s'éloigne de tous les chagrins qui m'attendent; il rentre dans le port, et je reste dans la tempête. Recommandez-le à tous les gens que j'ai déjà nommés, enfin à Mr. le Maréchal lui-même. Je ne me consolerais pas de la peine qu'on ferait au meilleur des hommes, et je croirais pas ma place honorable si on ne traitait pas mon devancier avec honneur. ${ }^{89}$

86 En 1785, l'Evêque d'Arras était Louis, François, Marc, Hilaire de Conzié (1732-1804). Ce prélat appartenait à une ancienne famille de Savoie. Son père avait été tué dans un accident de chasse par le Dauphin Louis, père de Louis XVI, ce qui lui avait valu, comme à ses frères, la protection royale. Après avoir été officier, il entra dans les ordres et devint Evêque de St-Omer en 1766 puis d'Arras en 1769. Président-né des Etats d'Artois, il concentra entre ses mains toute l'autorité de cette Assemblée et mena son diocèse militairement. Il se joignit au clergé et à la noblesse d'Artois pour refuser en 1789 toute représentation du Tiers-Etat aux Etats-Généraux. Il fut un des premiers à émigrer et s'attacha au Comte d'Artois (futur Charles X). Il fut mêlé à Edimbourg à toutes les intrigues des émigrés. (D'après la Grande Encyclop.)

87 Madame de Lauzun, épouse du Duc qui prit plus tard le nom de Biron et qui fut guillotiné en 1793, était Amélie de Boufflers, parente du Chevalier à un degré que nous n'avons pas pu définir.

88 Le Maréchal de Castries, successeur depuis 1780 au Comte de Sartines au ministère de la marine.

${ }^{89}$ Les extraits des lettres du Chevalier de Boufflers ont été faits par nous dans l'ouvrage de L. de Magnin et Henri Prat, Correspondance inédite de la Comtesse de Sabran et du Chevalier de Boufflers - 1778-1788 (Paris, Plon, 1875). 


\section{VII}

\section{LES DERNIERS JOURS DE LOUIS DE REPENTIGNY}

Très gravement atteint dans sa santé, Louis de Repentigny comprenait qu'il ne pourrait plus jamais reprendre de fonctions actives, mais il était en droit d'espérer qu'en haut lieu on aurait à cœur de récompenser les services qu'il avait rendus pendant une carrière commencée au sortir de l'enfance. Il crut pouvoir adresser au ministre de la marine, le 20 septembre 1786, une lettre que sa main défaillante, comme l'atteste une signature tremblée, le contraignait à faire écrire par une autre personne:

Monseigneur,

Daignez, s'il vous plait, jetter les yeux sur le Mémoire ci-joint que j'ai l'honneur de vous adresser. Si les motifs qu'il présente vous paraissent assez puissants pour m'accorder ce que je demande et que ma situation m'oblige de solliciter de la bonté du Roy et de la Votre, il ne me restera, Monseigneur, qu'à faire des vœux pour que ma santé me permette un jour de renouveller (sic) à Sa Majesté l'offre de mes services et de tâcher de mériter la continuation de ses grâces et de votre confiance.

Je n'ai pas fait mention dans ce Mémoire, Monseigneur, du grade de Maréchal de Camp auquel j'aurais été promu en 1781 si l'on ne m'avait retranché sept années de service en tems de guerre en qualité de brigadier, mais ce que je me dois à moimême m'oblige de ne pas abandonner les droits que me donnent à ce rang l'ancienneté et la nature de mes services, et je vous supplie d'avoir la bonté de les faire mentionner dans le brevet de mon traitement.

Si vous ne croyez pas, Monseigneur, que mes services me rendent susceptible (de recevoir) le Cordon Rouge ${ }^{90}$ que j'ai l'honneur de demander par

${ }^{90} \mathrm{Ne}$ sachant pas ce que Louis de Repentigny entendait par le Cordon Rouge, nous nous sommes adressé à Mr. Henri Torre, conservateur du Musée de la Légion d'Honneur qui a bien voulu nous répondre ce qui suit: \& Je pense qu'il s'agissait de la décoration de Commandeur de l'Ordre de St-Louis qui se portait comme celle de Grand-Croix (grand cordon porté en écharpe). La seule différence consistait en ceci que les Commandeurs, à la différence 
mon Mémoire, je vous supplie de daigner suppléer à cette grâce par celle du brevet de Maréchal de Camp, à prendre rang. ${ }^{91}$ En toute autre circonstance, je regarderais celà comme une grâce, mais de celle où je suis, c'est, Monseigneur, une justice que je réclame, et je le fais avec d'autant plus de confiance que j'ai l'honneur de la demander à un Ministre dont toutes les actions sont fondées sur ses principes, qui se fait un devoir de ne la refuser à personne et qui me l'a rendue à moi-même.

A l'égard de mon traitement, ce que je vous supplie de vouloir bien m'accorder ne sçaurait altérer l'ordre établi pour celui des autres Gouverneurs parce que je suis dans un cas tout particulier d'exception. Au reste, Monseigneur, il est des moyens de me le procurer sans exciter de réclamation; celui entre autres que j'invoque avec le plus de confiance, c'est la gratification annuelle qui m'a été accordée par Sa Majesté et annoncée d'une manière on ne peut plus précise avec l'assurance d'une place supérieure par son Ministre.

Résumant la requête de Repentigny dans une note du 24 septembre destinée au ministre, un collaborateur de celui-ci rappelait que l'ancien Gouverneur du Sénégal avait déjà fait valoir que les fonctions remplies par lui en Afrique l'avaient obligé à faire des dépenses fort lourdes, qu'il avait perdu ses meubles et ses provisions dans le naufrage du navire qui les transportait, que son secrétaire s'était enfui en lui volant $12000 \mathrm{f}$., enfin que sa santé délabrée entraînait des frais considérables. En conclusion le rédacteur de la note estimait pour sa part que la demande de Mr. de Repentigny était «trop étendue », mais qu'en raison de son état de santé et des pertes qu'il avait subies, il paraissait équitable de lui allouer un secours et une gratification d'autant plus urgente qu'il n'avait rien perçu depuis le mois de janvier précédent.

des Grands-Croix, ne portaient pas de plaque sur l'habit et le manteau. * Nous tenons à exprimer ici à M. H. Torre notre gratitude de nous avoir procuré ce renseignement.

91 Cette expression visait une nomination pour la forme. Sans attribution d'emploi jusqu'au jour où se produirait une vacance. 
Le ministre se borna à inscrire de sa main sur la note: « En attendant qu'on puisse régler la retraite de Mr. de Repentigny, on lui enverra une somme de $6000 \mathrm{fr}$. en à-compte sur ce qui lui sera accordé. » ${ }^{92}$ Repentigny remercia le ministre par une lettre datée du 26 septembre, rédigée, comme la précédente, par une autre personne et y apposa une signature où l'on perçoit la marque d'un grand effort.

Treize jours plus tard, le 9 octobre 1786, il décédait dans le logement qu'il occupait à Paris, 164 Rue Montmartre, son fils, officier de marine étant présent dans la capitale.

\section{LE TESTAMENT DE LOUIS LE GARDEUR DE REPENTIGNY}

Grâce à la grande obligeance de $\mathrm{M}^{\mathrm{e}}$ Tétard, notaire à Paris, successeur de $\mathrm{M}^{\circ}$ Lormeau titulaire de l'étude dans laquelle Louis Le Gardeur l'avait déposé, nous avons pu prendre connaissance $\mathrm{du}$ testament de ce dernier. Nous en exprimons notre vive gratitude à $\mathrm{M}^{\mathrm{e}}$ Tétard. Au dit testament, daté du 4 octobre 1786, était joint un codicille établi quelques jours avant la mort du signataire.

92 « Sous l'Ancien Régime, et même bien au delà, a écrit Mr. Albert Duchêne, Directeur honoraire des Affaires Politiques au Ministère des Colonies, dans son ouvrage, Histoire des Finances Coloniales de la France, chapitre V, il n'y avait pas de droit à pension. Celle-ci n'était qu'un don gracieux de l'Etat, c'est-à-dire du Souverain. L'âge n'y était pour rien, du moins à lui seul; les services rendus, également... Inversement, en leur absence, on pouvait se montrer généreux jusqu'à la munificence... On pouvait en voir attribuer le bénéfice alors qu'on était en pleine jeunesse et que l'on était investi, peut-être pour de longues années, de fonctions bien rémunérées, par ailleurs... On pouvait, au su de tous et de façon parfaitement avouée «cumuler 》 une pension annuelle et un traitement d'activité, de même on pouvait fort bien superposer une pension à une autre ... (Enfin) la pension peut subir des variations de chiffres, même très importantes, à égalité de grade ou à égalité dans la durée des services ...

Citons maintenant des exemples. La plus importante des pensions que nous rencontrions au titre colonial est celle de Bellecombe, successivement commandant dans l'Inde, maréchal de camp, aide-major général de la Martinique et Gouverneur Général de St-Domingue $28.000 £ \ldots$ mais Andrault, comte de Langeron, également maréchal de camp, ancien commandant du régiment de Foix à St-Domingue, ne figure que pour une pension de $8540 £$, et Bory, chef d'escadre, ancien Gouverneur des Iles-sous-le-vent aux Antilles $9000 £$; le vicomte de Souilhac, chef d'escadre, ancien Gouverneur général des îles de France et Bourbon 10.000 f. Les officiers subalternes n'atteignent pas pour la plupart $2000 £$ et pour beaucoup la pension est inférieure à 1000 £. \$ 
Après une profession de foi catholique, le testateur déclarait vouloir être inhumé dans le cimetière de la paroisse St-Eustache « avec la plus grande simplicité possible et sans frais, voulant qu'il n'y ait à son convoi que quatre cierges jaunes ».

Il désignait comme exécuteur testamentaire, son oncle, ${ }^{93}$ l'abbé Liénard de Beaujeu, ${ }^{94}$ demeurant à la communauté de la paroisse de St-Sulpice à Paris, le chargeant très spécialement de remettre à son fils, Louis Gaspard de Repentigny, son seul héritier, tous ses papiers dont ce dernier et l'abbé de Beaujeu pourraient seuls prendre connaissance.

Louis de Repentigny, hors la généralité de ses biens qui revenaient à son fils, Gaspard, Lieutenant des Vaisseaux du

93 L'abbé Louis Joseph de Beaujeu n'était pas en réalité l'oncle de Louis de Repentigny, mais un parent de sa mère, Marie Catherine Juchereau. Comme il arrive souvent, il lui donnait le titre d'oncle en témoignage de l'affectueux respect qu'il avait pour lui étant son cadet de treize ans.

94 \& Le seizième Jour d'aoust de L'an mil sept cens huit a esté batisé Louis Joseph né Le dit Jour fils de Louis Lienard Ecuier Sr. de Beaujeu Lieutenant d'une Compagnie du détachement de La marine et de dame tereze Migeon son Epouse. Le parein a Eté Alexandre Joeph De Lestringand Ecuier Sr. De St Martin, Capitaine d'une Compagnie du détachement de La Marine, La Mareine Dame Gertrude Le Gardeur épouse de Jean batiste Celoron Ecuier Sr. De Blainville Capitaine au dit détachement. signés

\author{
Louis Liénard de Beaujeu \\ De Lestringand de St Martin \\ Gertrude Le Gardeur \\ PRIAT prêtre.»
}

Sa mère, Thérèse Migeon (1678-1748), était la fille de J.-B. Migeon de Branssat (1639-1693), originaire de Moulins en Bourbonnais, juge au bailliage de Montréal en 1677, elle avait épousé, en premières noces, en 1692 (à Montréal) Charles Juchereau de Saint-Denis (1655-1703) premier juge royal de Montréal (1693). Elle convola à Montréal, en 1706, avec Louis Liénard de Beaujeu (1682-1750), tige de la famille de ce nom, dont elle eut Louis Joseph L. de Beaujeu, né en 1708. Ce dernier, passé jeune en France, y fut ordonné, prit ses degrés de docteur en Sorbonne (1742-1746), y devint chanoine de Saint-Denis, confesseur ordinaire du Roi Louis XVI. Il décéda en février 1791, au Séminaire Saint-Sulpice de Paris.

Le second fils de Louis L. de Beaujeu et de Thérèse Migeon, Daniel (1711-1755), marié à Québec en 1737 à Elisabeth Foucault, s'est immortalisé en Amérique, par sa défense du Fort Duquesne (aujourd'hui Pittsburg), où il fut tué en $\mathbf{1 7 5 5}$.

La copie de l'acte de baptême de l'abbé L. J. de Beaujeu et les précisions tant sur lui-même que sur sa famille nous ont été obligeamment procurées par Mr. l'Archiviste de la Cour Supérieure de Montréal à qui nous tenons à offrir notre reconnaissance. 
Roi, ${ }^{95}$ prévoyait quelques legs au profit des bénéficiaires suivants :

Les pauvres de la paroisse St-Eustache,

Les Récollets ${ }^{96}$ de la maison du Faubourg St-Martin à Paris,

Les domestiques de $\mathrm{Mr}$. Maugras, ${ }^{97}$

Le portier de la maison dans laquelle il logeait,

Un nommé Santerre, nègre libre de Lille (sic) de France, ${ }^{98}$

Un certain Deslandes (?)

Le cocher de Mlle Landriève (?)

Le total de ces legs s'élevait à 1222 Livres.

L'inventaire auquel il fut procédé le 17 décembre, en présence d'hommes de loi, de l'abbé de Beaujeu et du fils du défunt, comporte dans sa liste des vêtements, du linge de corps, une montre, une tabatière en écaille ornée d'un médaillon, une croix de St-Louis, des éperons d'argent, des boucles de même métal pour souliers, diverses créances sur un certain nombre de personnes; les documents relatifs à ces dernières étaient contenus dans un coffret.

Succession bien modeste au total.

\section{NOTES COMPLEMENTAIRES}

\section{LOUIS LE GARDEUR DE REPENTIGNY AVAIT-IL LE DROIT DE PORTER LE TITRE DE COMTE ?}

Il nous faut tout de suite préciser qu'il n'en usa qu'in extremis, si l'on peut dire, et dans les deux dernières lettres

95 Louis Gaspard de Repentigny était descendu à l'Hôtel de Grèce, Rue du Bouloi. Est-ce le même que l'Hôtel du Calvados actuel au No 25 de la Rue (?)

${ }_{96}$ Les Récollets appartenaient à une congrégation fondée en Espagne au XVe siècle; ils s'étaient introduits en France où Henri IV leur donna sa protection et se répandirent ensuite au Canada.

07 Nous n'avons pas pu identifier cette personne qui fut peut-être l'ascendant de Gaston Maugras, littérateur et historien (1851-1927). M. Maugras devait être un ami personnel de Louis de Repentigny. L'inventaire des affaires de ce dernier indique qu'il avait mis à sa disposition une chambre de son appartement pour qu'il ne fût pas obligé de loger en garni. Mr. Maugras jouissait d'une appréciable aisance puisqu'il avait à son service une cuisinière, un joké (sic) et un palfrenier.

98 Lisez Ile de France (Maurice). La profession de ce noir n'est pas indiquée, peut-être était-il le domestique personnel de Louis de Repentigny qui lui légua outre une somme d'argent, une douzaine de paires de souliers, une veste et une culotte de basin et un Boverlet (?) avec son contenu (?). 
adressées par lui quelques jours avant sa mort. Aucun autre document n'en fait mention, même lorsqu'il est signé de sa main. Seul, le sieur Lamiral, soi-disant député du Sénégal près les Etats-Généraux, le lui attribue dans son ouvrage L'Affrique et le Peuple Affriquain.

On ne saurait retenir le fait que l'avis de son décès et de l'apposition des scellés sur son logement, paru dans le journal de Paris le 16 octobre 1786 (p. 1195), en ait fait état, sans doute sur la déclaration des personnes qui l'avaient assisté au moment de son décès; et encore bien moins la singulière idée qu'eut un magistrat canadien, le juge Baby, d'affirmer dans un article concernant son frère aîné, qu'il devint marquis après la mort de ce dernier, ce qui eût été bien surprenant puisque ce dernier titre serait revenu à son fils François-Xavier, qui ne s'attribua, sans doute de son propre mouvement, que le titre de vicomte.99 Aussi bien Pierre de Repentigny ne porta jamais que le titre de Chevalier. ${ }^{1}$

Nous noterons enfin que Louis Gaspard, fils de Louis de Repentigny, officier de marine démissionnaire (1753-1808), s'attribua, dans l'acte de baptême de son fils Théodore, né à Capesterre (Guadeloupe), le 13 août 1786, le titre de comte et fit de même dans l'acte de baptême de son troisième fils Antoine Camille, conféré à Tours (Indre et Loire) le 16 juillet 1789. Sur l'acte de baptême de son fils aîné, dressé à Capesterre le 15 mai 1785, il est seulement qualifié Chevalier Seigneur de Repentigny.

Mr. Meurgey de Tupigny, conservateur aux Archives de France, dont nous avons sollicité l'avis, a bien voulu nous faire savoir qu'il ne lui apparaissait pas que le titre de comte ait fait l'objet de lettres patentes d'un souverain français ou étranger; et que son impression est qu'il ne s'agissait en l'occurrence que d'un titre de courtoisie.

99 Voir la notice sur Pierre, Jean-Baptiste, François-Xavier Le Gardeur de Repentigny, en mars prochain.

1 On verra plus loin que l'écrivain canadien Sulte attribue lui aussi le titre de marquis à Louis Le Gardeur de Repentigny sous un portrait qui n'est d'ailleurs pas le sien! 


\section{PORTRAITS DE LOUIS LE GARDEUR DE REPENTIGNY}

Désireux de placer dans la bibliothèque de Sault-Ste-Marie un portrait de Louis Le Gardeur de Repentigny, le comité institué dans cette ville en vue d'ériger le monument commémorant le souvenir du fondateur et de l'ancien commandant du fort établi en ce point alors compris dans les limites de la Nouvelle-France, s'était adressé à un attaché de l'ambassade des États-Unis à Paris pour qu'il entreprît les recherches nécessaires. Ce fonctionnaire eut l'idée, puisque la famille Le Gardeur était originaire de Normandie, de demander au Journal de Rouen d'informer ses lecteurs de la mission dont il avait été chargé. L'appel fut entendu par $\mathrm{M}^{\text {1le }}$ Thérèse du Fayal qui, semble-t-il, était une descendante de Mr. de Marchais, époux de la veuve du fils de Louis Le Gardeur de Repentigny, Louis Gaspard, ancien officier de marine, décédé à la Pointe-à-Pite, Guadeloupe, le 2 juillet 1808. Une photographie du portrait peint à l'huile dont $M^{11 \mathrm{le}}$ du Fayal était en possession fut ainsi envoyée à Sault-Sainte-Marie. Sur notre requête, Miss Myrtle A. Elliot a bien voulu nous en transmettre une reproduction.

A première vue, l'authenticité de ce portrait nous parut contestable pour les motifs suivants:

Tout d'abord, il était assez singulier qu'un souvenir de famille aussi précieux soit passé dans la famille du second mari de la veuve de Louis Gaspard de Repentigny alors que ce dernier avait lui-même un fils. Aussi bien Louis de Repentigny n'avait pas mentionné ce portrait dans son testament, et l'inventaire dressé après sa mort n'en fait pas état.

D'autre part, un examen attentif du tableau nous incitait à supposer qu'il était antérieur à la maturité de Louis de Repentigny. Consulté à ce sujet, Mr. Germain Bazin, conservateur en chef du département des peintures et dessins au Musée du Louvre, a eu l'obligeance de soumettre la photographie que nous lui avions communiquée au service d'Étude et de Documentation du musée. Celui-ci a estimé que l'œuvre avait certainement été exécutée avant 1730 , c'est-à-dire à une date à laquelle Repentigny n'était âgé que de neuf ans, et qu'elle pourrait être placée dans 
l'entourage de Rigaud, à moins que ce ne fût qu'une copie d'après une peinture de cet artiste. De toute façon, il serait permis de se demander comment Louis de Repentigny aurait pu supporter financièrement la dépense entraînée par l'exécution de son portrait par un peintre de mérite.

Nous n'insisterons pas sur un autre portrait prétendu de notre héros et que l'on a cru devoir insérer dans la brochure de Mr. Clever Bald, The French Seigniory at Sault Ste Marie. ${ }^{2}$

Cette gravure faite d'après un portrait trouvé dans l'ouvrage de Sulte: Histoire des Canadiens français, représente un personnage porteur d'une perruque datant manifestement du $\mathrm{XVII}^{\mathrm{e}}$ siècle; et l'auteur a cru pouvoir ajouter au nom de Louis Le Gardeur le titre de Maréchal (?) et au-dessous Marquis de Repentigny - Gouverneur du Sénégal !

Il est fâcheux que Sulte n'ait pas indiqué l'ouvrage d'où il avait extrait cette gravure ni les documents sur la foi desquels il avait attribué à Louis de Repentigny les titres de Maréchal et de Marquis.

\section{JUGEMENTS PORTÉS SUR LOUIS LE GARDEUR DE REPENTIGNY}

Deux personnes seulement ont porté des jugements défavorables sur Louis Le Gardeur de Repentigny. La première fut le marquis de Bouillé, du point de vue militaire. Nous avons fait ressortir plus haut les motifs de l'hostilité de ce haut personnage à l'encontre du Colonel commandant le Régiment de la Martinique et démontré la partialité méprisante dont ce dernier fut l'objet de son chef. La seconde fut le Sieur Lamiral dans deux publications. Le soi-disant député du Sénégal aux Etats-Généraux, ${ }^{3}$ celui-ci a mentionné dans son: "Plan d'Administration du nouveau régime pour tous les établissements français de la Côte d'Afrique », des scènes scandaleuses d'un Gouverneur et du Commandant de la troupe, Mr. de Repentigny et Mr. Ladevèze

\footnotetext{
2 Voir note précédente.
3 Le Sénégal n'avait en effet pas été invité à envoyer un député à
Paris.

2 Voir note précédente.
3 Le Sénégal n'avait en effet pas été invité à envoyer un député à
Paris.
} 
se donnant en spectacle au public en s'injuriant de la manière la plus indécente, oubliant de préciser que ce Ladevèze, commandant du Bataillon d'Afrique, était notoirement connu pour sa violence et son mauvais caractère. ${ }^{4}$

Dans son ouvrage, L'Affrique et le peuple Affriquain, le même Lamiral « ancien agent de commerce en Afrique » passant en revue les récents chefs de la colonie, s'est exprimé ainsi : "Mr. le Comte de Rep ... a suivi (Eyries). C'était un bon homme, tel qu'il le fallait pour une colonie comme celle-là, où il fallait un chef humain et qui se mette à la portée de tout le monde. Malheureusement les hommes sont faibles et se laissent séduire; ils ne voient pas toujours le piège qu'on leur tend. La Compagnie (de commerce) l'avait d'abord vexé, ensuite elle a su le mettre dans tous ses intérêts, je n'ai pas besoin de dire par quels moyens. »

Cette venimeuse insinuation de la part d'une personne dont la plume n'a épargné personne, sauf le Chevalier de Boufflers, auquel il ne résista pas à donner néanmoins un coup de griffe, n'est, bien entendu, pas appuyée de la moindre preuve. La situation financière difficile de Repentigny après son rappel du Sénégal, prouve à elle seule que son intégrité était au-dessus de tout soupçon. ${ }^{5}$

A la page 10 de son «Plan d'Administration », Lamiral s'en prend encore à Repentigny: «A Mr. Dumontet succéda Mr. de Repentigny. Le premier avec l'esprit d'un scélérat; le second faible et intéressé, se laissa circonvenir par l'autre et adopta une partie de ses erremens. Celui-ci avait la tête bouillante, il aimait

4 Voir plus haut, note 82.

5 «Mr. de Boufflers, écrit Lamiral (Plan d'Administration p. 10), ayant une grande consistence (sic) personnelle, en a imposé par sa raison et sa modération autant que par son nom et par son rang. Nous n'avons plus vu se répéter ces scènes honteuses (Boufflers s'étant empressé de faire remplacer le major Ladevèze par Blanchot, son ancien subordonné au Régiment de Chartres, dont il avait apprécié les qualités et le dévouement à son égard. Note du rédacteur), mais les haines, les querelles intestines et les dépenses que ce gouverneur n'a sans doute pas pu empêcher malgré son autorité et son esprit conciliateur, ont eu libre cours. > 
beaucoup à faire et il accrut encore beaucoup les dépenses de la colonie sans aucune utilité pour le commerce de la Nation. ${ }^{6}$

En face de ces imputations malveillantes et de surcrô̂t erronées, nous n'avons relevé que des éloges sur Louis de Repentigny, tant sur son courage dans les combats, la fermeté avec laquelle il commanda diverses unités, la régularité de l'administration des corps de troupe placés sous ses ordres. Quant à sa valeur morale, il nous suffira de rappeler l'appréciation que portèrent sur lui les membres de la commission chargée de se prononcer sur les affaires du Sénégal: «il est un homme d'honneur ». Mais en tant que chef d'une colonie, la même commission le jugeait au contraire «impropre à l'administration ».

Comme à cette époque, le Gouverneur des tetablissements de la Côte d'Afrique ne possédait, du point de vue civil, à peu près aucune charge administrative au sens que nous attribuons aujourd'hui à ce terme, il convient de définir, ce que la commission entendait par là. Si on se reporte à la liste des questions que cette assemblée fut appelée à examiner, ce que l'on reprochait à Repentigny, était d'avoir manqué au Sénégal, d'autorité sur son personnel, carence qui s'était particulièrement manifestée au cours de l'enquête concernant son prédécesseur. Nous ne reviendrons pas sur l'opinion que nous avons exprimée plus haut sur l'erreur que l'on avait commise en haut lieu en chargeant un successeur d'enquêter sur un prédécesseur et qui plaçait nécessairement le premier dans un grand embarras. Il est néanmoins possible qu'ayant pris de l'âge et déjà atteint dans sa santé, Louis de Repentigny ait manqué d'énergie pendant son séjour au Sénégal, encore qu'il s'y soit montré actif. Il est assez significatif à ce propos que Boufflers se préparant à rejoindre son poste écrivait au ministre sans le connaître: "Tout ce que

${ }^{6} \mathrm{D}$ 'après ses propres dires exprimés dans son livre, L'Affrique et le Peuple Affriquain, Lamiral était arrivé au Sénégal en 1779, un peu avant la reprise de St-Louis par Lauzun, en qualité d'Agent de la Cie de la Guyane pour tenter de développer la traite des noirs. Il aurait contribué à la réoccupation de St-Louis par ses travaux sans les préciser ce qui laisse un assez fort doute sur ce qu'il prétend, et avoir été chargé d'une mission importante sur laquelle il se garde de fournir le moindre détail. Vraisemblablement imbu d'idées rétrogrades et compromis par ce qu'il avait écrit à propos de la traite des noirs, il disparut prudemment après 1791 . 
j'apprends sur son compte annonce le meilleur et le plus brave homme du monde. »

Nous ne reviendrons pas sur les autres appréciations favorables portées sur lui par son successeur au Gouvernement du Sénégal, relevées plus haut par nous, et nous terminons en reproduisant celle du Directeur de la Compagnie du Sénégal: «C'était un homme sage, instruit et sans prétentions. Je lui fut particulièrement recommandé par $\mathrm{Mr}$. de Vergennes, ${ }^{7}$ ministre des Affaires Etrangères, son parent et son ami. ${ }^{8}$ Le ministre de la Marine, Mr. de Castries, parlait avantageusement de moi dans une lettre dont j'étais porteur pour Mr. de Repentigny. Il (ce dernier) me reçut avec bonté, avec distinction; il m'accorda sa confiance; nous vécûmes dans la plus parfaite harmonie et je n'ai eu qu'à me louer de ses dispositions pour le bien du service. »

\section{VIII}

\section{DESCENDANCE DE LOUIS DE REPENTIGNY}

S'il n'est pas assuré que $\mathrm{M}^{\mathrm{me}}$ Louis de Repentigny rejoignit son mari à la Martinique quand il fut nommé commandant du régiment de cette île, il est au contraire certain qu'elle ne l'accompagna pas en France quand il fut rappelé par le ministre ni plus tard, encore que cinq mois après sa nomination au poste de Gouverneur du Sénégal qu'il s'apprêtait à rallier, Repentigny ait demandé au ministre «de bien vouloir donner des ordres à l'Administration de la Guadeloupe en vue du retour de sa femme en France ainsi que de deux domestiques blancs et la femme de chambre qui l'avait accompagnée aux Antilles ».

7 Vergennes (Charles Gravier, Comte de) 1717-1787. Après une longue et belle carrière dans le service diplomatique, fut nommé par Louis XVI en 1776, ministre des Affaires Etrangères et en 1783, chef du Conseil Royal des Finances.

$8 \mathrm{Je}$ ne sais sur quoi se fondait Léonard Durand pour avancer que le comte de Vergennes était parent et ami de Louis de Repentigny; parent, certainement pas; ami, c'est fort douteux; peut-être avait-il entendu quelqu'un faire son éloge. 
Il est probable que ce furent des raisons de santé qui retinrent $\mathrm{M}^{\mathrm{me}}$ Louis de Repentigny à la Guadeloupe où elle décéda le 9 août $1784 .{ }^{\circ}$

Son neveu François Joseph Chaussegros de Léry, futur Baron de l'Empire, alors Ingénieur militaire à la Guadeloupe, seul de ses parents qui se trouvât près d'elle (son fils officier de marine étant en mer), écrivait à sa mère le 27 mai 1785 :

Il y a longtemps que je m'attendais à vous annoncer une mauvaise nouvelle et que je m'apprêtais à pleurer avec vous sur la mort de ma respectable tante de Repentigny. Mes regrets sont une suite de ses qualités qui la rendaient chère à tous ceux qui la connaissaient. C'est une mère de douleur expirant avec tout le sang-froid possible, ne regrettant la vie que par la privation où elle était alors de son fils et de son mari. Le départ de son mari pour le Sénégal lui avait porté le dernier coup... Personne autre que moi ne connaissait ses chagrins. Il y avait trop longtemps qu'elle était dans un état de souffrance morale pour tenir plus longtemps. ${ }^{10}$

De son côté, dans une lettre non datée, Louis de Repentigny annonçait au destinataire de cette correspondance qu'il avait perdu sa femme «victime de sa sensibilité sur tout ce qu'il éprouvait depuis quatre ans ».

Louis de Repentigny et Marie-Madeleine Chaussegros de Léry n'eurent qu'un fils, enfant unique, Louis Gaspard. Né à

9 Acte de décès de Mme Louis de Repentigny: L'an mil sept cent quatre vingt quatre, le dix du mois d'août dame Marie Magdelaine Regis CHAUSSEGROS DE LERY épouse de Messire Louis LE GARDEUR DE REPENTIGNY, brigadier des armées du Roy, gouverneur général du Sénégal et dépendances, décédée hier à onze heures du matin à l'habitation de messire LE PREVOT DUQUESNEL, agée de cinquante quatre ans, dix mois et quelques jours la dite dame native du Canada a été inhumée dans le cimetière de cette paroisse en présence de messire Alexis de PLUVIERS de Saint Michel, Louis Jacques Gabriel Marie HURAULT, sieur de Manoncour, Alexandre de Malartic, chevalier de l'ordre royal et militaire de Saint Louis et Louis Bernard BOUIRE, major de milices, habitans de cette paroisse qui on signé avec nous. Le chevalier de Saint Michel, HURAULT DE MANONCOUR, MALARTIC GIRAUUD DE CHARBONNIERES, BOUIRE, frère J. Fr CORDIER précheur curé.

10 Pierre-Georges Roy, La famille Chaussegros de Léry (Lévis, 1934), 40 pages in 80 - page 8. 
Québec le 10 juillet 1753, il entra très jeune dans la Marine militaire. Ayant atteint le grade de Lieutenant de Vaisseau, il obtint en 1790 un congé pour vaquer à ses affaires et partit pour la Guadeloupe. Il y participa activement avec le général Richepance à la lutte contre les esclaves révoltés non seulement dans cette île mais aussi à la Martinique. D'après le Gouverneur Ernouf: "il rendit de son bras et de ses conseils tous les services qu'on avait droit d'attendre d'un militaire et d'un colon ». Ayant demandé sa retraite, il ne fut pas compris dans la réorganisation de 1792 . Le $1^{\text {er }}$ novembre 1807 , il obtint un brevet provisoire de Capitaine de Port à la Pointe-à-Pitre, fonction qu'il ne devait remplir que peu de mois puisqu'il mourut dans cette ville le 2 juillet 1808.

De son mariage avec $\mathrm{M}^{\text {lle }}$ Marie-Madeleine Marguerite LePrévost Duquesnel, fille d'Antoine Jean-Baptiste Le Prévost, Chevalier, Seigneur Duquesnel, et de Marie-Madeleine, Gabrielle, Rose Giraud Duployé, de la Guadeloupe, ${ }^{11}$ naquirent trois fils: Louis François né à Capesterre le 24 septembre 1781, ${ }^{12}$ Théodore,

11 D'après l'historien canadien Pierre-Georges Roy (La famille Chaussegros de Léry (Lévis, 1934, 8) Mlle Leprévost Duquesnel, créole, aurait apporté à son mari une dot de douze à quatorze cent mille Livres.

12 Extrait des registres de l'Etat-Civil de la paroisse de Capesterre (Ile Guadeloupe) pour l'année mil sept cent quatre vingt cinq: L'an mil sept cent quatre vingt cinq, le 15 mai j'ai baptizé Louis François de REPENTIGNY, né le 24 septembre mil sept cent quatre vingt un, ayant été ondoiée le dit jour par le chirurgien Girard PEIRAFIT, fils en légitime mariage de messire Louis Gaspar LEGARDEUR chevalier, seigneur de Repentigny, lieutenant des vaisseaux du Roy, chevalier de l'ordre royal et militaire de Saint Louis et de dame Magdelaine Margueritte LEPREVOST DUQUESNEL, le parrain a été François Joseph CHAUSSEGROS DELERY, écuyer, ingénieur ordinaire du Roy en garnison à la Guadeloupe, la marraine a été Magdelaine Pauline Francoise HURAULT DE LIGNY veuve de messire d'OREL comte DESCORNAYS, garde de la Marine, demeurante à Changy paroisse Sainte Hyacinthe de la Capesterre, isle Guadeloupe. En foy de quoi nous avons signé le présent extrait en présence du père de l'enfant et avec le parain et la maraine.

DUQUESNEL DE REPENTIGNY, CHAUSSEGROS DE LERY, frère Berryer cordelier curé de la Petite Goyave, LE GARDEUR DE REPENTIGNY, DE LIGNY DES CORNAIS, DUQUESNE, HURAULT DE MANDREDOURT. 
né à Capesterre le 15 juin $1786^{13}$ et Antoine Camille né à Tours le 15 juillet $1789 .^{14}$

Louis-François mourut à Capesterre le 5 mai $1820,{ }^{15}$ étant chef de Bataillon en non activité et semble-t-il célibataire. Nous n'avons pas pu savoir ce que devint Théodore né à Capesterre le 15 juin 1786; les recherches faites par $\mathrm{M}^{\text {He }}$ Ménier aux Archives de la France d'Outre-Mer n'ont pas permis de retrouver l'acte de son décès à Capesterre; peut-être décéda-t-il jeune. Antoine Camille né à Tours partit à une époque non déterminée pour la

13 Extrait des registres de l'Etat-Civil de la commune de la Capesterre (Ile de la Guadeloupe) pour l'année mil sept cent quatre vingt six: L'an mil sept cent quatre vingt six et le traize aouts j'ai baptisé Théodore, né le quinze juin de la même année, fils en légitime mariage de messire Louis Gaspar LEGARDEUR compte de Repentigny et de dame Madelaine Pauline Marguerite DUQUENEIL le parrain a été Louis François sieur LEGARDEUR DE REPENTIGNY et la marraine Madelaine Pauline Françoise contesse de Carnays représentante dame Gille LANDRIENRE, le père et la marraine ont signé avec moi, le parrain n'a su cause de son bas âge.

DE LIGNY, DESCORNAIS, LE GARDEUR REPENTIGNY, frère LAY R/ desservant.

14 Copie de l'acte de naissance de Antoine Camille de Repentigny: Le seize Juillet mil sept cent quatre vingt neuf a été par nous curé soussigné baptisé Antoine Camille né d'hier fils de messire Louis Gaspard le Gardeur comte de Repentigny chevallier de l'ordre royal et militaire de St Louis, officier de la marine, et de dame Magdeleine Paulline marguerite Le Prevost du Quesnel son épouse légitime; a esté le parain le sieur Theodore le Gardeur, chevalier de Repentigny frère du baptisé, representé par M. Antoine Qilles Landrieves des Bordes cousin issu de germain du baptisé, paroisse de Saint Venant, la maraine dame Marguerite françoise Le Prevost du Quesnel épouse de messire Mithon comte de Genouilly, chevalier de St Louis et de Cincinnatus, chef d'escadre, sa tante demeurante à Montargy, representée par madame Magdeleine Paulline françoise Hurault de Ligny comtesse des Cornays sa cousine issue de germaine de cette paroisse qui a signé avec nous ainsi que le père présent et le representant. Signé: Hurault de Ligny ctess des Cornais, de Landriève Desborde, Le Gardeur Cte de Repentigny, Cuisnier Curé chne de St Venant.

15 Extrait des registres de l'Etat-Civil de la commune de la Capesterre (île de la Guadeloupe) pour l'année mil huit cent vingt.

L'an mil huit cent vingt, le cinq mai à huit heures du matin par devant noud Valentin Joseph CAIGNAT officier civil du quartier de la Capesterre, Lile Guadeloupe, sont comparus messieurs Jean Claude PRUDEAU, agé de quarante huit ans, habitant et Jean Bourgoin Valery agé de vingt neuf ans, négociant, tous deux résidants au dit quartier, lesquels nous ont déclaré que le quatre du présent mois était décédé sur l'habitation dite CHANGY à deux heures du matin, monsieur Louis LEGARDEUR DE REPENTIGNY agé de trente neuf ans, chef de bataillon non en activité ont les dits déclarans signé avec nous lè présent acte les jours, mois et an que dessus après lecture faite. PRUDEAU BOURGOIN VALERYCAIGNAT. 
Guadeloupe où il géra le domaine familial. Il décéda à Capesterre le $1^{\text {er }}$ avril 1820, cinq semaines après son frère Louis-François.

Antoine Camille de Repentigny avait épousé à Capesterre le 19 avril 1814, Aimée de Ganteaume ${ }^{16}$ dont les parents paraissaient avoir habité précédemment la Martinique, et dont il eut trois enfants: Louise Pauline, née à Capesterre le 17 janvier 1815, restée célibataire vraisemblablement; Lisette Antoinette et Joséphine Lucrèce, jumelles, nées à Petit-Bourg le 15 novembre 1819. Le nom de Repentigny, faute de descendants mâles, disparut ainsi de cette branche. ${ }^{17}$

$$
\text { L. JORE, }
$$

Gouverneur général hre de la France d'Outre-mer, Ancien gouverneur du Sénégal et dépendances.

(En mars prochain paraîtra la notice sur Pierre, JeanBaptiste, François-Xavier Le Gardeur de Repentigny, frère aîné de Louis Le Gardeur de Repentigny).

$16 \mathrm{La}$ famille de Ganteaume était peut-être alliée à celle de l'Amiral Ganteaume (1755-1818). Après la mort de son premier mari, Aimée de Ganteaume épousa Edme, Jean-Joseph Mignard qui mourut à Petit-Bourg en 1836; puis en troisièmes noces, Pierre Cabernot, dit Monpezat, originaire de Navarrens, dans les Basses-Pyrénées.

17 Lisette Antoinette épousa à Petit-Bourg le 24 octobre 1836, Pierre Charles, Murville Beaufils et n'aurait pas eu de descendance. Au contraire, Joséphine Lucrèce mariée à Petit-Bourg, le 20 octobre 1840 à Cyprien Clavie, originaire de St-Esprit (Basses-Pyrénées, commune aujourd'hui rattachée à Bayonne) en 1803 , donna le jour à un fils et à trois filles, tous nés à Petit-Bourg. 